\title{
Binary Power Control in Dynamic Spectrum Access
}

\author{
Brage Ellingsæter*† and Torleiv Maseng* \\ Norwegian Defense Research Establishment (FFI)*, email:torleiv.maseng@ffi.no \\ University Graduate Center at Kjeller $(\mathrm{UNiK})^{\dagger}$, email:brage@unik.no
}

\begin{abstract}
In future wireless networks there is a need for distributed power allocation algorithms which are simple, but at the same time yields sufficient spectral efficiency. In this paper we investigate binary power control schemes in a wireless multiuser, multiband interference system, where each user is restricted to use only one frequency band. We explore different binary power control schemes, and show how knowledge of the other users can be used to construct a utility function which embodies the well being of others. Simulation results show that binary power control yields significant increase in sum rate compared to water filling with number of users $\geq \mathbf{2}$, and in some scenarios yield sumrates above those achieved with much more complex algorithms.
\end{abstract}

\section{INTRODUCTION}

Over the years more and more services have been offered by wireless communications, and with these wireless services comes the demand for even more wireless services. It is now apparent that spectrum resources are scarce and that static spectrum allocation leads to significant under usage of the spectrum. Cognitive radio and more recently dynamic spectrum access has been posed as solutions, where the idea in dynamic spectrum access is that radio devices in need of spectrum obtains it on a dynamic basis.

Such radio devices must be able to adapt their transmission parameters and be able to exploit different parts of the spectrum. One of the key problems in terms of dynamic spectrum access and increased spectrum efficiency, is the problem of power allocation among different radio devices. In a dynamic spectrum access system, with multiple radio devices in the same area, it is likely that their spectral resources are the same. The power allocation problem is now that of a multiuser, multiband interference system.

The achievable rate region for all users in such a system is not generally known. In addition maximizing the joint system capacity is a non-convex optimization problem with regard to transmit power and is known to be NP hard [1]. But for same cases the optimal power allocation is known. For very low crosstalk and low power, equal power across all frequency bands is the optimal power allocation because log is a concave function. In [2] it was found that independent of transmit power, FDMA is optimal for high crosstalk gains. In general, the point where FDMA is optimal depends on both crosstalk gains and transmit power [2]. In addition to these fundamental limits, it was found that with imperfect knowledge of interference levels at the receiver, the power allocation converges to FDMA [3].

It can be argued that FDMA should also be used in scenarios where FDMA is not optimal. This is due to the low complexity and the fact that if all users are restricted to FDMA there is no incentive for users to change power allocations and therefore the algorithm does not need to converge to Nash equilibrium [1]. But in a practical system, there might not be as many available channels as users, making our definition of FDMA allocation impossible.

However, instead of selecting a channel which is not used by any other user, we can require a user to only allocate power to one channel, according to some criteria. By this restriction, the resulting power allocation scheme can be as simple as FDMA, in the case of best signal-to-interference-plus-noise ratio (SINR), or more complex depending on the algorithm. In terms of sum-rate, a radio link can be introduced in the network if the sum-rate over all links increases when this link is established. Even in the case of single channel power allocation this is not a trivial problem, and in this paper we simplify by only considering binary power control.

Due to the simplicity of binary power control, the subject has been extensively studied in both uplink and downlink [4] as well as the Gaussian interference channel [5] [6]. However, all these works has only considered systems where only one frequency band is available. Thus the comparison is made against adaptive power control. In cognitive radio systems, there might be more frequency bands available and therefore binary power control should be compared against algorithms which exploit the multiplexing gain of using more bands. This is done in this paper, and we investigate optimal binary power control, as well as simpler forms of binary power control and compare them in terms of sum-rate against other well known algorithms which utilize the multiplexing gain.

\section{System Model AND Fundamental Limits}

\section{A. System Model}

We consider a wireless communication system in which $N$ radio links share a common bandwidth of $K$ orthogonal frequency bands, or channels. Each radio link is thought of as a user, where each user consists of transmitter and desired receiver, i.e. user $i$ consists of transmitter $i$ and receiver $i$. The set of users $1, \ldots, N$ is denoted $\Omega_{N}=\{1, \ldots, N\}$. Similarly, the set of channels is denoted $\Omega_{K}=\{1, \ldots, K\}$. Two or more users transmitting on the same channel are subject to interference from each other, and this interference is treated as white Gaussian noise.

Channel gains from transmitter $i$ to receiver $j$ over channel $k$ is denoted $h_{i j}(k)$. We assume each receiver suffers from the same thermal noise, denoted $N_{0}$. This thermal noise is 
modeled as additive white Gaussian noise. The joint capacity of this system is given as

$$
C=\sum_{i \in \Omega_{N}} \sum_{k \in \Omega_{K}} \log _{2}\left(1+\frac{\left|h_{i i}(k)\right|^{2} p_{i}(k)}{N_{0}+\sum_{j \in \Omega_{N}, j \neq i}\left|h_{j i}(k)\right|^{2} p_{j}(k)}\right)
$$

Since each user can only transmit on one channel, the transmit power constraint for each user is given as

$$
0 \leq p_{i}\left(k^{*}\right) \leq P_{i, \max } .
$$

where $k^{*}$ is the channel user $i$ has chosen to transmit on.

We assume the transmitter is able to obtain knowledge of the noise plus interference levels from the receiver through some medium, e.g. a feedback channel. Through this medium we also assume the transmitter is able to estimate the channel gain between itself and the desired receiver. With this information the transmitter can estimate the effective noise plus interference level in each channel as

$$
N I(k)=\frac{1}{\left|h_{i i}(k)\right|^{2}}\left(N_{0}+\sum_{j \in \Omega_{N}, j \neq i}\left|h_{j i}(k)\right|^{2} p_{j}(k)\right)
$$

\section{B. Fundamental Limits}

In the previous section we introduced some fundamental limits of the system model considered, based on results found in [2]. In addition to the limits found in [2] we can state some fundamental limits and properties for the case where the power constraint of each user approaches infinity. Note that these limits only hold when interference is treated as noise and there is no structure in the interference (interference alignment).

Theorem 1. In a system with $N$ users, $K \geq N$ channels and non-zero interfering channel gains, FDMA is the optimal power allocation when $P_{i, \max } \rightarrow \infty \forall i \in \Omega_{N}$.

Proof: With out loss of generality (WLOG) we can assume a system with 2 users and 2 channels. Each user has power $P$. Next assume that each user has allocated a portion $t_{l} \neq 0,1, l=1,2$ of its power to channel 1 and $\left(1-t_{l}\right)$ to channel 2. WLOG we can ignore all channel gains. The total rate of the system then becomes:

$$
\begin{aligned}
R & =\log \left(1+\frac{P t_{1}}{N_{0}+P t_{2}}\right)+\log \left(1+\frac{P\left(1-t_{1}\right)}{N_{0}+P\left(1-t_{2}\right)}\right) \\
& +\log \left(1+\frac{P t_{2}}{N_{0}+P t_{1}}\right)+\log \left(1+\frac{P\left(1-t_{2}\right)}{N_{0}+P\left(1-t_{1}\right)}\right)
\end{aligned}
$$

with $P \rightarrow \infty, R$ reduces to

$$
\begin{aligned}
\lim _{P \rightarrow \infty} R & =\log \left(1+\frac{t_{1}}{t_{2}}\right)+\log \left(1+\frac{\left(1-t_{1}\right)}{\left(1-t_{2}\right)}\right) \\
& +\log \left(1+\frac{t_{2}}{t_{1}}\right)+\log \left(1+\frac{\left(1-t_{2}\right)}{\left(1-t_{1}\right)}\right)<\infty .
\end{aligned}
$$

Assuming FDMA

$$
\lim _{P \rightarrow \infty} R=\lim _{P \rightarrow \infty} \frac{1}{2} \log \left(1+\frac{P}{N_{0}}\right)+\frac{1}{2} \log \left(1+\frac{P}{N_{0}}\right)=\infty .
$$

From this theorem we have the following corollaries

Corollary 2. In a system with $N$ users, $K \geq N$ channels and non-zero interfering channel gains, FDMA is the optimal power allocation when $N \rightarrow \infty$.

Corollary 3. In a system with $N$ users, $K=1$ channels and non-zero interfering channel gains, if all $N$ users transmit at the same time the sum-rate $R \rightarrow 0$ as $N \rightarrow \infty$, due to the interference.

From the second corollary we can conclude that with a high number of users in the same area, maximum sum-rate can not be achieved when all users transmit at the same time. I.e some user have to have $p_{i}(k)=0 \forall k \in \Omega_{K}$ to achieve maximum sum-rate.

\section{BINARY POWER CONTROL}

To find the optimal power allocation that maximizes sumrate is still a hard problem even though we restrict a given user to only use one channel. To simplify the problem, we only consider binary power control in this paper. In general, binary power control means that a transmitter can either transmit with power level $P_{\max }$ or $P_{\min }$. But from Corollary 3 we know that in a dense network some users must be silent to achieve maximum sum-rate and thus for a given user $i$ we have

$$
p_{i}\left(k^{*}\right)=\left\{\begin{array}{cc}
P_{i \max } & \text { if some criteria } \\
0 & \text { otherwise }
\end{array}\right.
$$

thus $P_{\min }=0$.

Considering only one available channel, [6] found that when the sum-rate can be approximated by the arithmetic geometric mean or in the low $S I N R$ regime $(\log (1+S I N R) \approx S I N R)$ binary power control is optimal. This motivates us to consider binary power control in our system model.

\section{A. Optimal Binary Power Control}

In terms of maximizing sum-rate, optimal binary power control means that a user is allowed to transmit on channel $k$ if the sum-rate over channel $k$ is increased by establishing the new link. If we number the users transmitting over channel $k$ as $1, \ldots, \tilde{N}$, and the new user as $\tilde{N}+1$, the the condition for transmission over channel $k$ for user $\tilde{N}+1$ is

$$
\sum_{i=1}^{\tilde{N}} \log _{2}\left(1+\frac{P_{i, \max }}{N I_{i}(k)}\right)<\sum_{i=1}^{\tilde{N}+1} \log _{2}\left(1+\frac{P_{i, \max }}{N I_{i}(k)}\right) .
$$

Deriving easy-to-evaluate expressions from this condition is hard. By assuming that the network is dense (i.e. number of users in a given area is large) and assuming that the SINR is either in the high regime $(\log (1+S I N R) \approx \log (S I R)$, where $S I R$ means neglecting the thermal noise) or in the low SINR regime $(\log (1+S I N R) \approx S I N R)$ the conditions for transmission simplifies to [4]:

$$
\begin{aligned}
& S I R>e \text { high SINR regime } \\
& S I R>1 \text { low SINR regime }
\end{aligned}
$$


The drawback is that these criteria for transmission are only optimal in these SINR regimes, and as we will show in the simulations, they are on average very suboptimal. Next, we will see how a binary power control scheme can be constructed with varying degrees of information.

\section{B. Only NI knowledge}

We will first explore the binary power control scheme with minimal knowledge, i.e. only knowledge of the effective noise plus interference $(N I)$ levels at the receiver at the transmitter. In Section II, $k^{*}$ was defined as the channel chosen for transmission. With only knowledge of the $N I$ levels, the candidate channel for transmission is given as

$$
k^{*}=\min (N I)
$$

where $\min (N I)$ returns the index of the channel with lowest noise plus interference level. Due to Corollary 3, we know that to achieve maximum sum-rate, not all users can transmit at the same time in a dense network. Therefore, the power will be allocated in channel $k^{*}$ according to

$$
p_{i}\left(k^{*}\right)=\left\{\begin{array}{cc}
P_{i \max } & S I N R\left(k^{*}\right) \geq \eta \\
0 & \text { otherwise }
\end{array}\right.
$$

where $\eta$ is some threshold that should be chosen to maximize sum-rate. As noted above, according to [4] the threshold $\eta$ should be 1 in the low SINR regime and $e$ in the high SINR regime, to achieve maximum sum rate.

\section{With Location and Power Level Knowledge}

If a transmitter also has information about the location of the other receivers and power vector of the other transmitters, it can construct its power control scheme to take into account the performance of other users.

Consider the scenario depicted in Fig. 1. Two cognitive radio links has established communication between their respective transmitters and desired receivers. Each link transmits on a different frequency band than the other. Next, there is a third cognitive radio that wants to establish communication between its transmitter and desired receiver. The receiver measures noise and interference. Since transmitter 1 is closer to receiver 3 than transmitter 2 , it is likely that there is more interference in frequency band 1 than in frequency band 2 .

Without location information and without regard for the performance of the other links, transmitter 3 would use frequency band 2. But with location information, it knows that receiver 1 is farther away than receiver 2 and in light of this information transmitter 3 should allocate power to frequency band 1 with regard to the other users.

To encourage such behavior we must construct a power allocation scheme which rewards the well being of other users. To do this, we construct a pay-off function, $U$, that embodies this property.

If we denote the rate achieved with $P_{i, \max }$ in channel $k$ as $R_{i}(k)$, the pay-off function is given as

$$
U_{i}(k)=R_{i}(k)-\sum_{j \in \Omega_{N}, j \neq i} w\left|h_{i j}(k)\right|^{2} P_{i, \max }
$$

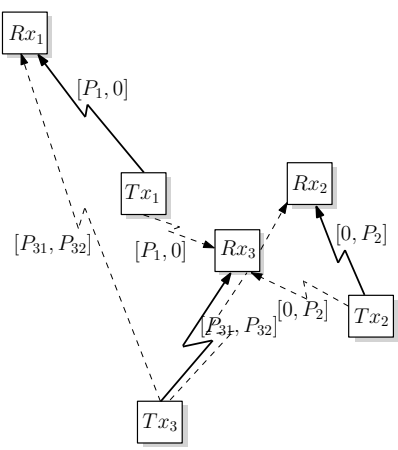

(a)

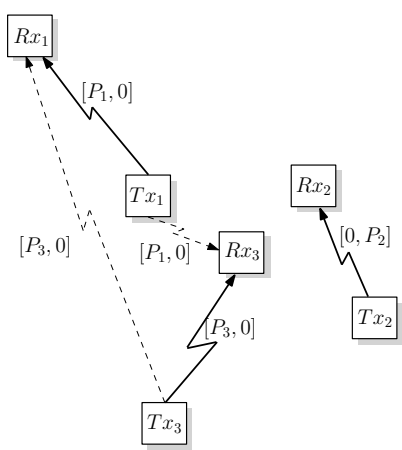

(b)
Fig. 1. Two cognitive radio links exists before radio link 3 tries to establish a radio link. The two established links transmit on two different channels, unfortunately there is only 2 available frequency bands. (a) The new transmitter observes the interference and must decide which channel to use. (b) Based on additional information, transmitter 3 knows that it will create the least interference if it uses frequency band 1 .

where $\left|h_{i j}(k)\right|^{2} P_{i, \max }$ is the interference created by user $i$ to user $j$ in channel $k$. If user $j$ does not transmit over channel $k$, the interference created is zero. $w$ is a scaling variable that must be chosen so that limiting interference to other users has the desired weight in the pay-off function. We do not consider individual user weights since the channel gain has this effect. As in the case of only NI information, we first find the candidate channel to transmit on, i.e.

$$
k^{*}=\max \left(U_{i}(k)\right)
$$

and user $i$ will allocate power in channel $k^{*}$ according to

$$
p_{i}\left(k^{*}\right)=\left\{\begin{array}{cc}
P_{i \max } & U_{i}\left(k^{*}\right) \geq \eta \\
0 & \text { otherwise }
\end{array}\right.
$$

From the definition of our pay-off function it is clear that we must know the channel gains between transmitter $i$ and all other receivers. To obtain accurate channel estimates would require substantial overhead, but with slow moving or static users, location information can be used to estimate the channel gains.

The drawback is that there will be fading from shadowing in the system, even with static users, which might affect the performance of our power allocation scheme.

\section{STABILITY CRITERIA}

Since this system is an interference system, there might be an incentive for a user to adjust its power allocation after another user has allocated power. This is why all power allocations in such a system is of an iterative nature. The goal of an iterative power allocation algorithm is to converge to a stable point where no user has the incentive to change its allocation. In terms of game theory, such a point is called a Nash equilibrium (NE).

Even though we have simple binary power control algorithms, it is still necessary for the power control to be stable and thus requires to reach a NE. We will briefly describe the conditions necessary for convergence to a NE. 
Lets denote a combination of users in the system as $\Omega_{C}$. A combination is a possible state in the system with a subset of users in each channel. If we denote user 1 as $u_{1}$, user 2 as $u_{2}$ and so on, the following are possible combinations, with 2 channels:

$$
C_{1}=\left[\begin{array}{c}
u_{1} \\
\emptyset
\end{array}\right], C_{2}=\left[\begin{array}{cc}
\emptyset & \\
u_{1} & u_{2}
\end{array}\right], C_{3}=\left[\begin{array}{l}
u_{1} \\
u_{2}
\end{array}\right] \ldots
$$

where each row is a channel and $\emptyset$ means the channel is empty.

Since a user can either allocate power to one channel or stay silent, the number of possible combinations of users is $(K+1)^{N}$. Thus we have $\Omega_{C}=\left[C_{1}, C_{2}, \ldots, C_{\left|\Omega_{C}\right|}\right]$ and $\left|\Omega_{C}\right|=(K+1)^{N}$. Clearly if no users in the combination has an incentive to change channels, the combination is table. However, if in $C_{2}$ in (16), $u_{1}$ wants to change channel after $u_{2}$ starts signaling on the same channel, $C_{2}$ is not stable.

We now denote the coexistence between a pair of users as $u_{1}(k) \leftarrow u_{2}(k)$ if $u_{2}$ can transmit in channel $k$ at the same time as $u_{1}$ and not have an incentive to change power allocation. If both can coexist with the other, we denote this as $u_{1}(k) \leftrightarrow u_{2}(k)$. We can now state the condition for the existence of a NE.

Theorem 4. A NE exists if $\exists C \in \Omega_{C}$, such that

$$
u_{i}(k) \leftrightarrow u_{j}(k), \forall i, j, k \in \Omega_{N} \cap \Omega_{C}
$$

In plain text this says that a NE exists if there exists a combination such that all users transmitting on channel $k$ can coexist with the other users transmitting on channel $k$, for all $k \in \Omega_{K}$.

Unfortunately, the existence of a NE does not automatically mean the NE is reached. In fact, if we assume $C_{1}$ is the combination that first occurs in time, $C_{2}$ the next, and so on, then if a combination equal to $C_{1}$ occurs later in time, then a $\mathrm{NE}$ can not be reached.

Theorem 5. A NE can not be reached if

$$
C_{j}=C_{i}, j>i+1
$$

Thus, although simple in theory, single band binary power control is not as trivial as seemed at first. The problem of stability is implemented as follows in this paper. We let each user have memory of its past power allocations, and the first user to suspect that it is in a loop, i.e. suspects that a NE can not be reached, shuts down transmission and waits one iteration. By doing this it hopes that once it starts transmitting again, the combination has changed sufficiently so that a NE can be reached. If the same user notices its in a loop again, it shuts down for 2 iterations, then 4,8 and so on. To be able to start transmitting again, the user also has to satisfy (12) or (15).

The upside is that once a NE is reached, this is the Pareto optimal solution [7] due to our definition of stability and criteria for transmission. In general a NE is not Pareto optimal [7].

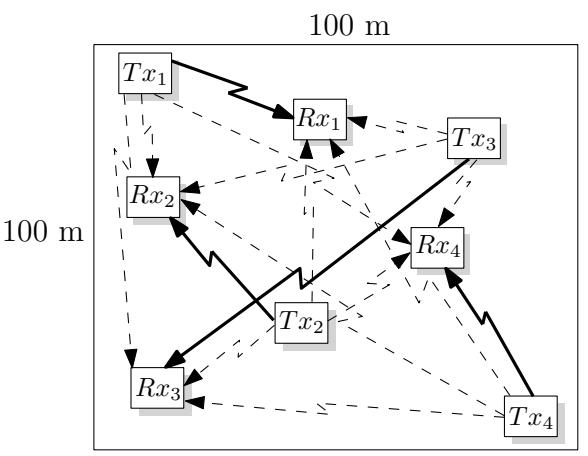

Fig. 2. Environment used for simulations in this paper. Users transmitters and desired receivers are uniformly distributed in an area of $100 \times 100$ meters.

\section{Simulation Results}

In order to investigate the performance of the proposed binary power control schemes in terms of sum rate and number of active users, we consider the wireless multiuser, multiband system given in Fig. 2. A user's transmitter and desired receiver is randomly placed in an area of $100 \times 100$ meters, with 5 available channels. In this paper we assume channel gains are only dependent on distance, i.e.

$$
\left|h_{i j}(k)\right|^{2}=d_{i j}^{-\gamma}, \forall k \in \Omega_{K}
$$

where $\gamma$, which is the path loss exponent, is set to 3.6 according to measurements given in [8] for an urban area. We assume each user has a transmit power constraint of $1 \mathrm{~W}$, and $N_{0}$ is set to $-70 \mathrm{dBm}$, so that a transmitter can reach a receiver on the opposite side of the area.

We compare the proposed power allocation schemes presented in this paper against the well known iterative water filling algorithm (IWFA)[1] and an algorithm known as PIWFA [9]. IWFA is a greedy algorithm where each user tries to maximize rate selfishly subject to the power constraint. PIWFA, on the other hand, is an algorithm which performs close to optimal in terms of sum rate, but as a consequence has added complexity [9].

The sum rate of the system with different amount of information is plotted in Fig. 3. For simplicity we have used the same $\eta$ with only NI knowledge and with additional information. In the plots we have chosen 3 different values for $\eta$ which yields 3 interesting cases. With $\eta=0$ (plotted with cyan color), only NI knowledge and low weighting (low $w)$ yields the same sum-rate, but by increasing the weight sufficiently the sum-rate can be increased by as much as 10 $\mathrm{b} / \mathrm{s} / \mathrm{Hz}$. With $\eta=3 e$ (plotted with green color), the weighting in the utility function (13), has less impact on performance, and it suffices to have $w \geq 1$. This is due to the fact that with high $\eta$, less users will satisfy the condition for transmission, even with low $w$. But as is seen, with 20 users there is a $18 \mathrm{~b} / \mathrm{s} / \mathrm{Hz}$ gain in performance by using the utility function, i.e. more knowledge. It is worth noticing that with only NI knowledge, sum-rate achieved with $\eta=3 e$ is significantly larger than the sum-rate achieved by the utility function and $\eta=0$. 


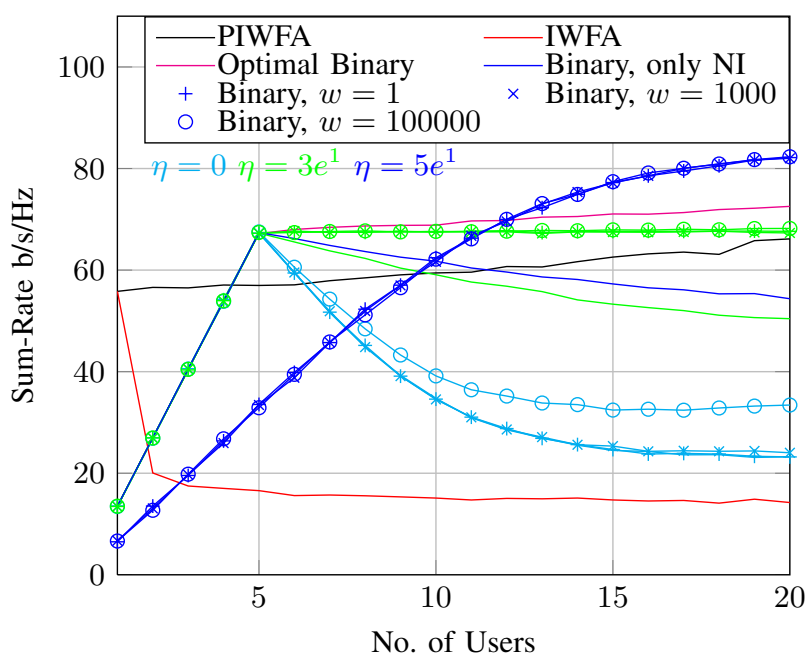

Fig. 3. Sum rate plotted against increasing number of users for binary power control.

The sum-rate achieved with $\eta=3 e$ and the utility function is just below the sum-rate achieved with optimal binary power control (plotted with magenta color). Optimal binary power control is found in the simulations by comparing the left hand side in (8) to the right hand side for each user in MATLAB.

With high number of users, sum-rate can be further increased by increasing $\eta$. With number of users equal to 20 , setting $\eta=5 e$ (plotted with blue color) yields a sum-rate 10 $\mathrm{b} / \mathrm{s} / \mathrm{Hz}$ larger than the one achieved with optimal binary power control. This may seem strange, but can be explained by noting that the optimal binary power control presented in this paper is in fact suboptimal. The reason is that we have not taken into account optimal user selection in this paper. As shown in [4], binary power control can be improved by optimally selecting those users allowed to transmit according to their channel gain between transmitter and desired receiver. This is called multiuser diversity gain. By making $\eta$ sufficiently large, this is actually what happens. With low number of users, i.e. sparse network, there is less probability that any user will satisfy a high $\eta$. However, in a dense network, there is a high probability that some users will satisfy this high $\eta$. This explains why the sum-rate increases more slowly while at the same time a higher sum-rate is achieved with high number of users for $\eta=5 e$.

All the results described above can be further explained by Fig. 4 and Corollary 3. Fig. 4 shows the number of active users divided by the number of available users in the system. To perform close to optimum, it is clear that PIWFA limits the number of active users so that roughly only 2 users are active at the same time. The effect of both increasing $\eta$ and $w$ is that the number of users transmitting is limited. With $\eta=0$ and only NI knowledge, and since $S I N R$ is nonnegative, all users are able to transmit. By increasing $\eta$, we can decrease the number of active users, and through weighting we can assure that only those users with high quality links are able to transmit.

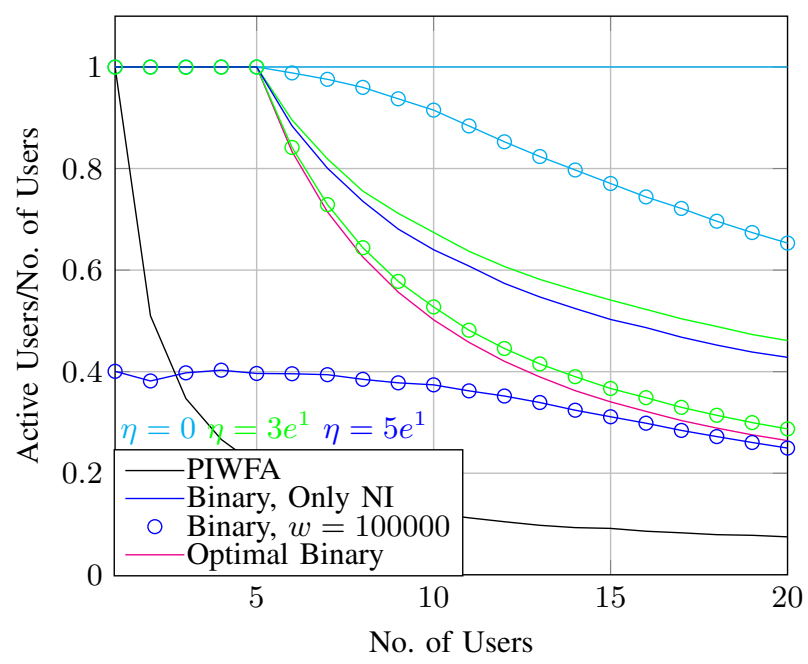

Fig. 4. Active users divided by total number of users for binary power control.

\section{CONCLUSION}

In this paper we have investigated binary power control schemes in a wireless multiuser, multiband interference system where a user is restricted to single channel transmission. We proved that in a dense network, only a subset of users can transmit in order to achieve maximum sum-rate. Different binary power control schemes were derived and the stability of the algorithm was investigated. Through simulations we found that constraining the users to only transmit on one channel and thus not exploiting the multiplexing gain only degrades performance in systems with more channels than users and that in a dense network single band binary power control can even improve the rate achieved with more complex algorithms (PIWFA) by sufficient settings in the algorithm.

\section{REFERENCES}

[1] W. Yu, "Competition and cooperation in multi-user communication environments," Ph.D. dissertation, Stanford University, 2002.

[2] S. Hayashi and Z.-Q. Luo, "Spectrum management for interferencelimited multiuser communication systems," Information Theory, IEEE Transactions on, vol. 55, no. 3, pp. $1153-1175,2009$.

[3] R. Gohary and T. Willink, "Robust iwfa for open-spectrum communications," Signal Processing, IEEE Transactions on, vol. 57, pp. $4964-4970$, dec. 2009.

[4] Z. Bassem, H. Majed, A. Hayara, and G. E. Oien, "Binary power allocation for cognitive radio networks with centralized and distributed user selection strategies," Physical Communication 1 (2008) 183-193, September 2008.

[5] A. Gjendemsjo, D. Gesbert, G. Oien, and S. Kiani, "Optimal power allocation and scheduling for two-cell capacity maximization," in Modeling and Optimization in Mobile, Ad Hoc and Wireless Networks, 2006 4th International Symposium on, pp. 1 - 6, 2006.

[6] A. Gjendemsjo, G. Oien, and D. Gesbert, "Binary power control for multicell capacity maximization," in SPAWC, pp. 1-5, 2007.

[7] M. J. Osborne, An Introduction to Game Theory. Oxford, U.K.: Oxford Univ. Press, 2004.

[8] A. Goldsmith, Wireless Communications. New York, USA: Cambridge University Press, 2005.

[9] F. Wang, M. Krunz, and S. Cui, "Price-based spectrum management in cognitive radio networks," Selected Topics in Signal Processing, IEEE Journal of, vol. 2, pp. $74-87$, feb. 2008. 